\title{
A Practical Design of TCSC Controllers for the Inter-area Transient Stability Control Using Real-Time Measurements
}

\author{
Chi-Shan Yu \\ Chih-Wen Liu \\ Department of Electrical Engineering, National Taiwan University, Taipei, Taiwan
}

\begin{abstract}
In this paper, an improved Optimal Aim Strategy (OAS) for the design of Thyristor Controlled Series Compensator (TCSC) controller has been proposed to enhance the inter-area transient stability of interconnected power systems. Due to the existence of some problems in applying conventional OAS to the power system transient stability control, some solutions have been proposed to improve the conventional OAS, and the analytic design procedures also have been proposed to achieve the desired performance. In addition, for multimachine inter-area transient stability OAS control, the reduced order model has been proposed to design the improved OAS command. The shaping of the reduced order model involves the real-time measurements which are available from Phasor Measurement Units (PMUs). Finally, some simulation studies are employed to test the controller. The results show that the controller is effective and robustive for damping transient stability swings under various conditions including severe faults and postufault change of network configuration.
\end{abstract}

Keywords : TCSC, Optimal aim strategy, transient stability

\section{I - INTRODUCTION}

For many years, a great deal of research interest has been focused on the study of the low frequency oscillation of interconnected power system. These oscillations also termed inter-area mode oscillations, have a typical frequency range of oscillation from 0.1 to $0.8 \mathrm{~Hz}$. These oscillations caused by severe faults also induce transient stability problems. There are some challenges in developing controllers for such problems. First, the nonlinear characteristic of the power system model due to large signal variations complicate the controller design. Secondly, in considering the centralized controllers, the large amount of system data transfer between each machine presents another challenge. Recent advances of high power electronic devices such as Flexible AC Transmission System Devices (FACTS) have provided a potential solution to transient stability control problems. The FACTS devices such as thyristor controlled series compensators (TCSC) have been proven their additional benefits of improving system damping. The TCSC device contributes variable impedance to the transmission lines. Due to the fast response, the TCSC is attractive to improve the transient response of the power system, especially the interarea dynamic response. Therefore, the TCSC is used in this paper to demonstrate proposed controllers.

There have been many proposed control strategies for improving transient stability of power systems. There are Fuzzy Control [1], and Neuro-network Control [2], Time Optimal Bang-Bang Control [3,4], Feedback Linearization Control [5], Optimal Aim Strategy Control [6,7]...etc. In considering these control strategies, the benefits of Fuzzy Control and Neuro-network are no need of knowledge of the system model. However, it takes much of time to train Fuzzy and Neuro-network controllers. The Time Optimal Bang-Bang Control could provide the fastest response. However, due to the open loop design and no consideration of the time delay in the controller, the time optimal bang-bang controller could not provide the acceptable results when the system equilibrium varies or the controller does not switch exactly at the desired time instant. As proposed in [3], the author uses the concept of energy function and switch band to solve these problems. However, the control strategy still could not provide enough damping as the system trajectory gets close to the equilibrium point. The Optimal Aim Strategy (OAS) $[6,7]$ is a powerful control strategy for nonlinear controller design. For many years, there have been few papers proposed for the OAS controller. Although the simple procedure in designing the OAS controller, there still have some problems while directly applying OAS controllers to power systems. For examples, the response time could not be specified in designing the OAS controller. In this paper, the Improved OAS (IOAS) controller has been proposed. The proposed controller could give some solutions to the above problems.

For decentralized control strategy, the observation decoupled state space (ODSS) model in [8] is proposed for power systems. Although the ODSS technique is effective for many power system control problems, it is not suitable for the proposed IOAS controller, since the changing equilibrium point of the ODSS model while computing the local equilibrium [8] makes the "aim" varying with time. In $[3,9,10]$, the reduced order model is presented for inter-area oscillation control, and this model is suitable for the proposed IOAS controller design. The shaping of the reduced order model uses the real-time measurements as feedback signals which are available from Phasor Measurement Units (PMUs). The only needed measurements while shaping this reduced order model are voltage phasors of the tie-line terminal voltage. Some simulation studies in this paper show that the controller is effective and robustive for damping transient stability swings under various conditions including severe faults and post-fault change of network configuration.

The paper is organized as follows. The power system model for single machine infinite bus system (SMIB) and the proposed reduced order model are presented in section II. In section III the design procedures of improved optimal aim strategy have been described. Some simulation studies are 
employed to test the controller in section IV. Finally, a brief conclusion is given in section $\mathrm{V}$.

\section{II - POWER SYSTEM AND TCSC CONTROLLER MODEL}

\section{A $\cdot$ Single machine infinite bus system (SMIB system)}

In this paper, the classical generator model has been used to design the proposed controller. Firstly, considering the single machine infinite bus (SMIB) system

$$
\begin{aligned}
& \dot{\delta}=\omega \\
& \dot{\omega}=\frac{1}{\mathrm{M}}\left(\mathrm{P}_{\mathrm{m}}-\frac{\mathrm{EV}}{\overline{\mathrm{U}}} \sin \delta\right)-\mathrm{D} \omega
\end{aligned}
$$

where, $\overline{\mathrm{U}}=\mathrm{x}_{\mathrm{L}}+\mathrm{x}_{\text {eq }}$

$$
\begin{aligned}
& x_{L}: \text { equivalent transmission line impedance. } \\
& x_{\text {eq }}: \text { equivalent impedance of TCSC }
\end{aligned}
$$

\section{$B \cdot$ Interconnected multimachine system}

The multimachine system considered in this paper can be divided into two areas interconnected by a relatively weak tie. Each generator in this system is modeled by the classical model. A TCSC has been placed in series with the transmission line to increase the power transfer capability, and to provide additional damping to the inter-area mode oscillation in this system.

In [3], one reduced model has been proposed for the multimachine inter-area mode oscillation and suitable for the proposed IOAS controller design. However, the full system states are needed to shape the centralized reduced system model. Since the wide spread of the real power system, the large data communication is not easy to implement. Moreover, the internal voltages of this model corresponding to the $\mathrm{COA}$ are not measurable variables. Therefore, there needs another reduced order model for illustration of the reduce order model, and the following variables should be defined first

$$
\begin{array}{ll}
M_{1}^{s}=\sum_{i=1}^{k} M_{i} & M_{2}^{s}=\sum_{i=k+1}^{n} M_{i} \\
\delta_{1}^{s}=\sum_{i=1}^{k} M_{i} \delta_{i} / M_{1}^{s} & \delta_{2}^{s}=\sum_{i=k+1}^{n} M_{i} \delta_{i} / M_{2}^{s} \\
\omega_{1}^{s}=\sum_{i=1}^{k} M_{i} \omega_{i} / M_{1}^{s} & \omega_{2}^{s}=\sum_{i=k+1}^{n} M_{i} \omega_{i} / M_{2}^{s}
\end{array}
$$

where indices $i=1 . . k$ refer to the generators in subsystem 1 , and indices $\mathrm{i}=\mathrm{k}+1 . \mathrm{n}$ denote generators in subsystem2. Therefore, two-machine equivalent of the inter-area dynamics is given by

$$
\begin{array}{ll}
\dot{\delta}_{\mathrm{i}}^{s}=\omega_{\mathrm{i}}^{\mathrm{s}} & , \mathrm{i}=1,2 \\
\dot{\omega}_{\mathrm{i}}^{\mathrm{s}}=\frac{1}{\mathrm{M}_{\mathrm{i}}^{\mathrm{s}}} \Delta \mathrm{P}_{\mathrm{ei}}^{\mathrm{s}} & , \mathrm{i}=1,2
\end{array}
$$

where $\quad \Delta P_{e i}^{s}=\sum_{j}\left(P_{m j}-P_{e j}\right), \begin{cases}j=1 . k & \text { for } i=1 \\ j=k+1 . n & \text { for } i=2\end{cases}$

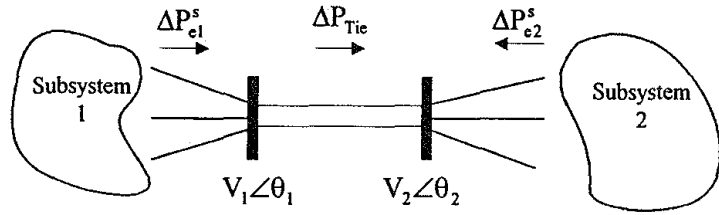

Figure 1 - real power variation of interconnected power system

$P_{m j}$ : the desired mechanical power input of machine $j$.

$P_{e j}$ : the real power output of machine $j$.

Considering the multimachine interconnected power system shown in Fig.1, and some reasonable assumptions are given as follows:

- Interconnected transmission line is lossless.

- The power system load is constant PQ load.

- The real power deviation $\Delta \mathrm{P}_{\mathrm{el}}^{\mathrm{s}}$ transmitting from the subsystem 1 to the interconnected transmission line is equal to the real power deviation $\Delta \mathrm{P}_{\mathrm{Tie}}$ transmitted between the interconnected transmission line.

- The real-power deviation $\Delta \mathrm{P}_{\mathrm{Tie}}$ transmitted between the interconnected transmission line is equal to the negative value of the real power deviation $\Delta \mathrm{P}_{\mathrm{e} 2}^{\mathrm{s}}$ transmitting from the subsystem 2 to the interconnected transmission line.

With these assumptions, the real-power deviation $\Delta \mathrm{P}_{\mathrm{ei}}^{\mathrm{s}}$ of the subsystem i could be substituted with $\Delta \mathrm{P}_{\text {Tie }}$, where

$$
\Delta \mathrm{P}_{\mathrm{Tie}}=\mathrm{P}_{\mathrm{spec}}-\frac{\mathrm{V}_{1} \mathrm{~V}_{2}}{\mathrm{X}_{\mathrm{L}}+\mathrm{X}_{\mathrm{eq}}} \sin \left(\theta_{1}-\theta_{2}\right)
$$

where $\theta_{1}$ and $\theta_{2}$ are the phase angles of the tie-line terminal bus voltages, $V_{1}$ and $V_{2}$ are the magnitudes of the terminal bus voltage, and $P_{\text {spec }}$ is the desired real-power transmission between the interconnected system in steady state.

From Eq.(5), the only needed measurements to implement the tie-line dynamics are the terminal bus voltage phasors at the both ends of the tie-line. Then, the dynamic equation of subsystem 1 is given as

$$
\begin{aligned}
& \dot{\delta}_{1}^{s}=\omega_{1}^{s} \\
& \dot{\omega}_{1}^{s}=\frac{1}{\mathrm{M}_{1}^{s}} \Delta \mathrm{P}_{\mathrm{Tie}}
\end{aligned}
$$

Now the inter-area dynamics between the two subsystems could be represented as:

$$
\begin{aligned}
& \dot{\delta}_{\mathrm{COA}}^{\mathrm{s}}=\omega_{\mathrm{COA}}^{\mathrm{s}} \\
& \dot{\omega}_{\mathrm{COA}}^{\mathrm{s}}=\frac{1}{\mathrm{M}_{1}^{\mathrm{s}}} \Delta \mathrm{P}_{\mathrm{Tie}}+\frac{1}{\mathrm{M}_{2}^{\mathrm{s}}} \Delta \mathrm{P}_{\mathrm{TiC}}
\end{aligned}
$$

where

$$
\delta_{\mathrm{COA}}^{\mathrm{s}}=\delta_{1}^{\mathrm{s}}-\delta_{2}^{\mathrm{s}} \quad \omega_{\mathrm{COA}}^{\mathrm{s}}=\omega_{1}^{\mathrm{s}}-\omega_{2}^{\mathrm{s}}
$$

From the network structure, generator 1 to $\mathrm{k}$ of subsystem 1 construct a coherency group and generator $k+1$ to $n$ of subsystem 2 construct another coherency group. The dynamic 


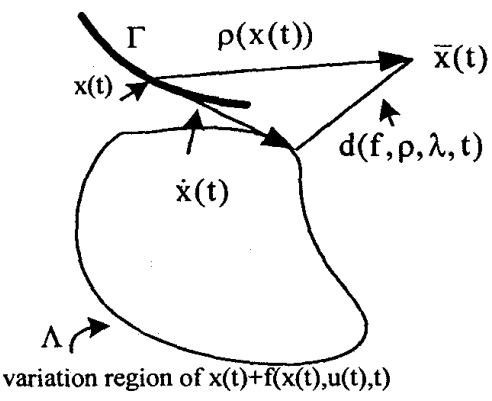

Figure 2 - Optimal aim strategy

behavior of $\theta_{12}=\theta_{1}-\theta_{2}$ could be represented as $\dot{\theta}_{12}=\dot{\delta}_{12}^{s}+\varepsilon$, where $\varepsilon$ is a small random variable. Thus, the dynamic behavior of the interconnected multimachine system could be approximately represented as the following reduced order model

$$
\begin{aligned}
& \dot{\theta}_{12}=\omega_{\mathrm{COA}}^{\mathrm{s}} \\
& \dot{\omega}_{\mathrm{COA}}^{\mathrm{s}}=\frac{1}{\mathrm{M}_{1}^{\mathrm{s}}} \Delta \mathrm{P}_{\mathrm{Tie}}+\frac{1}{\mathrm{M}_{2}^{\mathrm{s}}} \Delta \mathrm{P}_{\text {Tie }}
\end{aligned}
$$

Obviously, the above equations only involve the tie line terminal bus voltage phasors, which could be measured by PMUs. Furthermore, the controller design using the reduced order model could have almost the same effects on the interarea mode dynamics of the multimachine power system, as the controller design using Eq.(7).

The reduced order model proposed in this paper is only for the controller design. While in time-domain simulation for testing the TCSC controller, the original classical model of interconnected multimachine system is used.

\section{III - THE PROPOSED TCSC CONTROLLER}

\section{$A \cdot$ Optimal-aim strategies (OAS)}

The basic principle of the OAS is illustrated in Fig.2. Considering the system trajectory $\Gamma$, the OAS command is designed to guide the system state $x(t)$ to always move toward the system equilibrium state $\bar{x}(t)$. If the command is bounded to limit the variation of state deviation in the region of $\Lambda$, then $\dot{x}(t)$ shown in Fig.2 is preferable to reach the equilibrium state $\bar{x}(t)$. The direction vector toward the object is

$$
\rho(x(t))=\bar{x}(t)-x(t)
$$

, and the velocity vector of system state is

$$
\lambda(t)=\dot{x}=f(x(t), u(t), t)
$$

, and the difference between the two vectors is measured by

$$
\mathrm{d}(\mathrm{f}, \rho, \lambda, \mathrm{t})=\|\rho(\mathrm{x}(\mathrm{t}))-\lambda(\mathrm{t})\|_{2}
$$

, where \|\|$_{2}$ is the 2 -norm. Therefore, the OAS is to design an input $u(t)$ to minimize Eq.(12).

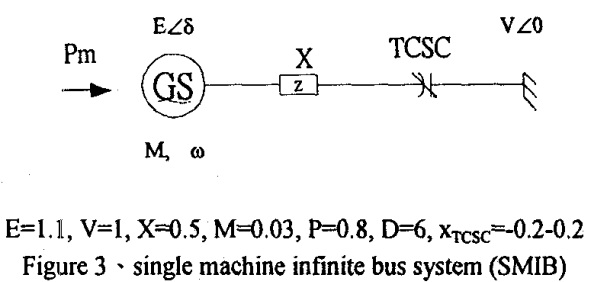

In reference [4], the author presented the following OAS controller command based on the above principle: state equation:

$$
\dot{x}(t)=f(x(t), t)+B u(t),
$$

controller command:

$$
u(t)=r\left(K B^{T}(\bar{x}(t)-x(t)-f(x(t), t))\right.
$$

where $K=\left(B^{T} B\right)^{-1}, r$ is a function to give the constrain to limit the controller output. Using Eq.(13) for SMIB, one can obtain the OAS TCSC controller command as follows: state equation:

$$
\begin{aligned}
& \dot{\delta}=\omega \\
& \dot{\omega}=\frac{1}{\mathrm{M}}\left(\mathrm{P}_{\mathrm{m}}-\frac{E V}{\overline{\mathrm{U}}} \sin \delta\right)-\mathrm{D} \omega
\end{aligned}
$$

controller command:

$$
\begin{aligned}
\overline{\mathrm{U}} & =\mathrm{x}_{\mathrm{L}}+\mathrm{x}_{\mathrm{eq}} \\
& =\mathrm{r}\left\{(\mathrm{EV} \sin (\delta)) /\left[\mathrm{P}_{\mathrm{m}}+\mathrm{M}(\omega-\mathrm{D} \omega)\right]\right.
\end{aligned}
$$

The SMIB system shown in Fig. 3 is employed for testing the performance of TCSC controllers using optimal aim strategy and improved optimal aim strategy, respectively. We try to gain some insights from SMIB system. Then we can extend the results to interconnected multimachine system. A 3-phase fault followed by 5 cycles faults clearing and reclose of circuit breaker is assumed in SMIB system. Fig.4 shows the time response of $\delta(t)$ of SMIB using OAS TCSC controller.

\section{B 、 Improved Optimal Aim Strategy (IOAS)}

It is observed in Fig. 4 that OAS TCSC controller is able to damp out oscillation by faults. However, there exists steadystate errors using OAS TCSC controller. The main reason of the existence of the steady-state errors is that the OAS TCSC controller command has no feedback information of $\delta$. Thus, unless the controller gain is very large, the steady-state errors could not vanish. In this section, we introduce one simple method to improve this point.

The improved OAS controller command for SMIB is designed as follows:

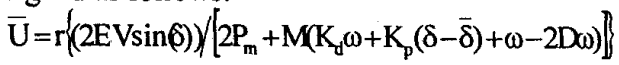

where $K_{p}$ and $K_{d}$ are constants to be designed.

For systematic design of parameters $K_{p}$ and $K_{d}$, we substitute the improved controller command into the system 
state equation Eq.(1), one can obtain a simple result as follows:

$$
\left[\begin{array}{c}
\dot{\delta} \\
\dot{\omega}
\end{array}\right]=\left[\begin{array}{cc}
0 & 1 \\
-\frac{1}{2} \mathrm{~K}_{\mathrm{p}} & -\frac{1}{2}\left(\mathrm{~K}_{\mathrm{d}}+1\right)
\end{array}\right]\left[\begin{array}{l}
\delta \\
\omega
\end{array}\right]+\left[\begin{array}{l}
0 \\
\frac{1}{2}
\end{array}\right]\left(\mathrm{K}_{\mathrm{d}} \bar{\omega}+\mathrm{K}_{\mathrm{p}} \bar{\delta}\right)
$$

It is observed that Eq.(18) is a pure linear state equation. If $K_{p}$ and $K_{d}$ are chosen, all the parameters of the linear state equation are known. The behavior of the nonlinear system is just equivalent to a linear one by adding the improved OAS TCSC controller. Therefore, one can use the pole placement method or the LQ optimal methods to design parameters $\mathrm{K}_{\mathrm{p}}$ and $\mathrm{K}_{\mathrm{d}}$. Moreover, all signals involved in Eq.(17) are available from PMUs.

In this paper, for ease of illustration, the pole placement technique has been used to design parameters $K_{P}$ and $K_{d}$. The designed parameters are $K_{p}=304, K_{d}=44$, the poles of closedloop system are $-16.67 \pm 8.04 \mathrm{i}$. Simulation results are shown in Fig.5. Comparing Fig.5 with Fig.4, the performance of IOAS TCSC controller is better than that of OAS TCSC controller in terms of settling time and steady-state error.

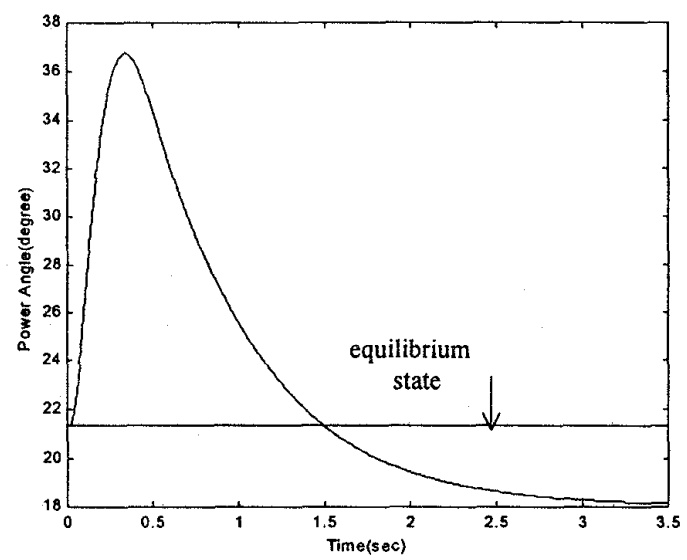

Figure 4 - time response of $\delta$ of SMIB using OAS TCSC controller after a 3phase fault

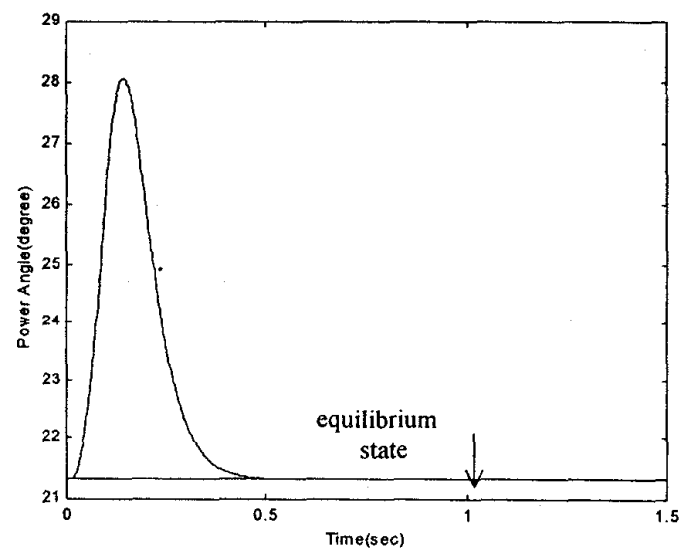

Figure 5 - time response of $\delta$ of SMIB using IOAS TCSC controller after a 3-phase fault
The design process of the IOAS TCSC controller is very easy and straightforward by proper choosing parameters $K_{p}$ and $K_{d}$, then one can get the desired response. Due to the linearity of the closed-loop system, the linear control theory could be easily applied.

\section{IV - SIMULATION RESULTS}

In this section, the reduced order modeling techniques and IOAS TCSC controller developed in section II and III are applied to a interconnected multimachine system shown in Fig.6. Due to existence of inter-area mode and intra-mode oscillation at the same time, the test system are always used to demonstrate the performance of controllers of the multimachine system. The parameters are as follow:

- Subsystem 1: $\mathrm{P}_{\mathrm{m} 1}=1.0, \mathrm{P}_{\mathrm{m} 2}=1.5, \mathrm{P}_{\mathrm{L} 5}=0.5, \mathrm{P}_{\mathrm{L} 6}=1.0$, $\mathrm{x}_{16}=0.05, \mathrm{x}_{26}=0.05, \mathrm{x}_{56}=0.25$,

- Subsystem 2: $\mathrm{P}_{\mathrm{m} 3}=1.5, \mathrm{P}_{\mathrm{m} 4}=1.0, \mathrm{P}_{\mathrm{L} 7}=1.5, \mathrm{P}_{\mathrm{L} 8}=2.0$, $\mathrm{x}_{47}=0.05, \mathrm{x}_{38}=0.05, \mathrm{x}_{78}=0.25, \mathrm{~b} / 2=0.15$

- Tie-line : $\mathrm{x}_{67}(1)=\mathrm{x}_{67}(2)=2.5, \mathrm{x}_{67}(3)=4$.

All the above units are in per-unit. The classical generator model and constant PQ load model are used, where the generator inertia constant $\mathrm{H}_{1}=\mathrm{H}_{4}=3$ p.u. and $\mathrm{H}_{2}=\mathrm{H}_{3}=5$ p.u. . Compensation range of TCSC are $x^{\max }=1.5$ p.u. $(60 \%$ compensation $), \quad x^{\mathrm{min}}=0.1$ p.u. $\quad(4 \% \quad$ compensation $)$ and equilibrium operating point $\bar{x}=0.5 p \cdot u$. ( $20 \%$ compensation). The reactance of the fix capacitor is $x^{\mathrm{fc}}=0.5 \mathrm{p} . \mathrm{u} . \quad(20 \%$ compensation).

Many simulation studies, under a wide variety of system and fault conditions, have been conducted on the test system. Due to space constraint, the following two kinds of fault are considered.

- Temporary fault : Three phase fault occurs near the Bus6. The fault is cleared after four cycles and transmission line remains in service.

- Permanent fault: Three phase fault occurs at the first transmission line between Bus6 and Bus7. Fault cleared after four cycles, and transmission line is disconnected. According to the proposed IOAS design procedures, the pole placement technique is used to obtain parameters $\mathrm{K}_{\mathrm{p}}=20.58$, $K_{d}=10.11$, such that the poles of closed-loop system are $2.78 \pm 1.6 \mathrm{i}$. The response of temporary fault is shown in Fig.7, and the response of permanent fault is shown as Fig.8. In Fig.7, for demonstration of the effects of proposed reduced order model, the star line is designed using the proposed reduced order model, and circle line is designed using Eq.(7). The simulation results show that the power angle response of the reduced order model is almost the same as that using the model Eq.(7). Therefore, the proposed reduced order model is suitable for designing the multimachine controller. Moreover, the simulation results also show that the proposed IOAS TCSC controller are effective and robustive for damping transient stability swings under various conditions including severe faults and post-fault change of network configuration. Furthermore, the IOAS TCSC controller command is 


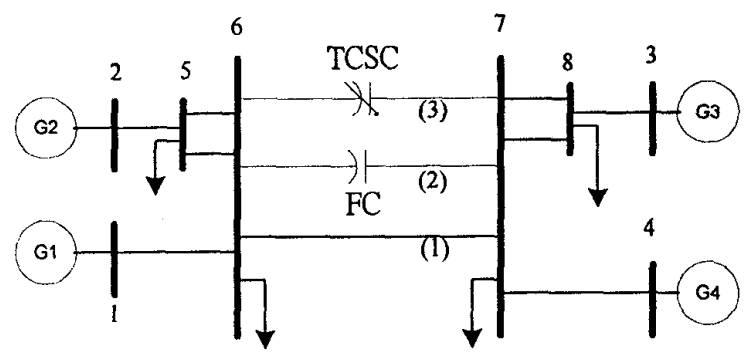

Figure 6 - 4-machines, 8-bus test system

continuous and smooth, and will not cause the severe change in the transmission line impedance such as the Bang-Bang TCSC controller command. From Fig.7 and Fig.8, it is observed that the proposed IOAS TCSC controller has the potential to control inter-area transient stability swings using real-time measurements.

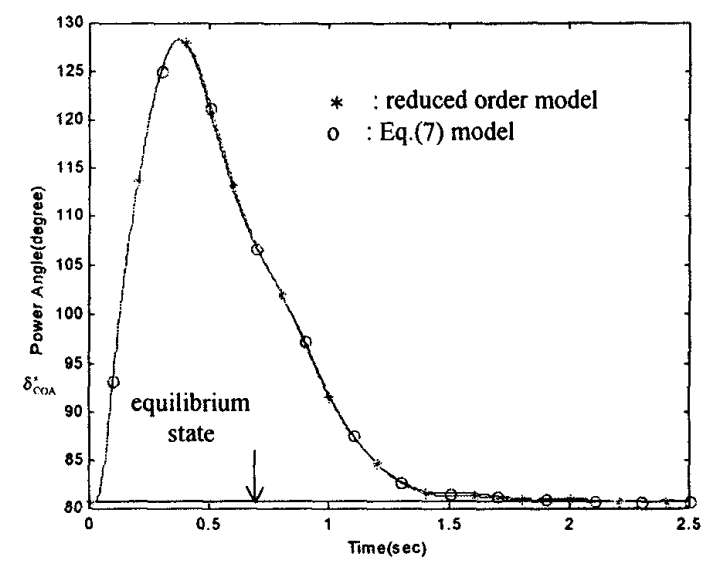

Fig.7 - time response of $\delta_{\mathrm{COA}}^{\mathrm{s}}$ of multimachine using IOAS TCSC controller after temporary fault

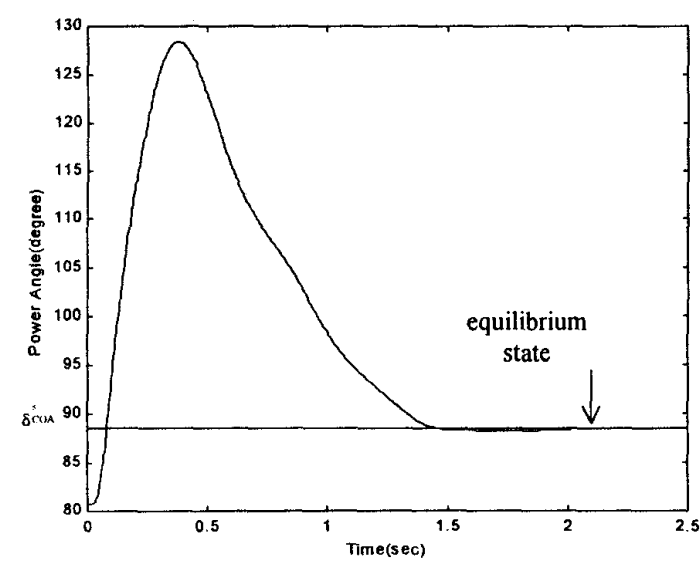

Fig. 8 - time response of $\delta_{\mathrm{COA}}^{s}$ of multimachine using IOAS TCSC controller after permanent fault

\section{$\mathrm{V} \cdot$ CONCLUSION}

In this paper, the improved optimal aim strategy (IOAS) has been proposed to improve some problems of the conventional OAS in application to the power system. For the multimachine control, the reduced order model has also been proposed in this paper. The shaping of the reduced order model involves the real-time measurements which are available from phasor measurement unit (PMU). The simulation results also show that the proposed IOAS TCSC controller and reduced order model are effective and robustive for damping transient stability swings of multimachine system under various conditions including severe faults and post-fault change of network configuration.

\section{VI 、 REFERENCE}

[1] HUYAMA, T., and KUGIMIYA, M. "Advanced PID type fuzzy logic power system stabilizer," IEEE. Trans. EC-9, pp.514-520 (1994).

[2] ZHANG, Y., MALK, O. P., HOPE, G. S., and CHEN, G. P., "Application of an inverse input/output mapped ANN as a power system stabilizer," IEEE. Trans. EC-9, pp.433-441 (1994)

[3] DMITRY, N., KOSTEREV, and WOJTEK, J. KOLODZIEJ, "BandBang series capacitor transient stability control," IEEE. Trans. PWRS, vol. 10, no. 2, pp.915-924 (1995).

[4] JAEWON CHANG, and JOE H. CHOW, "Time-optimal series capacitor control for damping inter-area modes in interconnected power systems," IEEE., Trans., PWRS., vol. 12, no 1, pp.215-221, (1997).

[5] Y. L. TAN, and Y. WANG, "Robust nonlinear design for transient stabilization using series power flow compensator," I. J. EPES., vol. 19, no. 6, pp.367-374 (1997)

[6] BARNARD, R. D. "Optimal-aim control strategies applied to largescale, nonlinear regulation and tracking system," IEEE. Trans., CAS-23, pp.800-306, (1976)

[7] X.Y.LI, Y.H.SONG, X.C.LIU, and J.Y.LIU, "Nonlinear optimalvariable-aim strategy for improving multimachine power system transient stability," IEE. Proc-Gener. Transm. Distrib., vol.143, no.3, pp.249-252, (1996).

[8] ZABORSZKY, J., WHANG, K.W., PRASAD, K., and KATZ, I.N. "Local Feedback Stabilization of Large Interconnected Power Systems in Emergencies," Automatica, vol.17, no.5, pp. 673-686, (1981).

[9] M. PAVELLA, P.G.MURTHY, Transient Stability of Power Systems, JOHN WILEY (1994)

[10] PETER W. SAUER, and M. A. PAI, Power system dynamics and stability, Prentice-Hall, Inc. (1998).

\section{BIOGRAPHIES}

Chi-Shan Yu was born in Taipei, Taiwan in 1966. He received his B.S. and M.S. degree in electrical engineering from National Tsing Hua University in 1988 and 1990, respectively. $\mathrm{He}$ is a candidate for the Ph.D. degree in the electrical engineer department at National Taiwan University, Taipei, Taiwan. His current research interests are FACTS controller and transient stability analysis. 


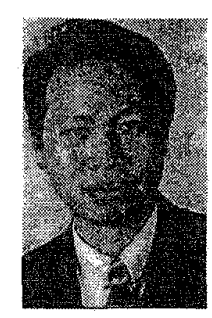

Chih-Wen Liu was born in Taiwan in 1964. He received the B.S, degree in Electrical Engineering from National Taiwan University in $1987, \mathrm{Ph} . \mathrm{D}$. degree in electrical engineering from Cornell University in 1994. Since 1994, he has been with National Taiwan University, where he is associate professor of electrical engineering. His research interests include application of numerical methods to power system, motor control and GPS time transfer. 